essential but still emerging workforce, not only in high-income countries but also worldwide.

\section{RF-405 OCCUPATIONAL SKIN DISEASE SURVEILLANCE USING A CLINICAL PATCH TEST DATABASE OVER TIME: THE RESULTS OF THE NORTH AMERICAN CONTACT DERMATITIS GROUP: 2001-2016.}

${ }^{1} D$ Linn Holness, Joel DeKoven, Benjamin DeKoven, NACDG Members. 'University of Toronto, Canada and St Michael's Hospital, Canada

\subsection{6/OEM-2021-EPI.368}

Introduction Clinical databases may provide useful information on occupational diseases. Occupational skin diseases are common. Patch testing is performed as part of the diagnostic process for contact dermatitis. There are several large, pooled patch test databases that track results over time. The North American Contact Dermatitis Group is a group of dermatologists in the United States and Canada who use standardized methods for patch testing and reporting and undertake regular analysis of their pooled results.

Objectives Using NACDG data, to examine the diagnoses, common workplace allergens and trends over time for workrelated allergens.

Methods Data from North American Contact Dermatitis Group (NACDG) datasets from 2001-2016 were analyzed to determine the frequency of occupationally relevant allergic patch test reactions to a screening tray of allergens and examine trends over time. NACDG members record diagnosis, work-relatedness and industry and occupation using the United States 1990 Census Bureau codes. Descriptive analysis of workers with occupational skin disease was performed using standard statistical tests; logistic regression was used to examine trends over time.

Results Of 38,614 patients tested, 4471 (11.6\%) had occupationally-related skin disease; $70.5 \%$ of individuals with occupationally-related skin disease had a final diagnosis of allergic contact dermatitis. Fifty one percent were male and the median age was 43 . The most common occupationally related agents were rubber accelerators (carba mix, thiuram mix, diphenylguanidine), Bisphenol A epoxy resin, formaldehyde, methylisothiazolinone and metals (nickel sulfate hexahydrate and potassium dichromate). Over the 16 year period there was a significant increase in occupationally relevant responses to carba mix and methylchloroisothiazolinone/methylisothiazolinone and a decrease in 2-mercaptobenzothiazole.

Conclusion Ongoing analysis of pooled patch test databases provides information about the common work-related allergens that can be used to inform prevention activities. Examining trends over time can identify particular allergens for more careful assessment of exposures and help target prevention efforts.

\section{RF-412 SURVEILLANCE OF COVID-19 CASES AMONG MEDICAL LABORATORY STAFF IN SOUTH AFRICA}

${ }^{1}$ Kerry Wilson, David Jones, Nonhlanhla Tlotleng, Felix Made, Graham Chin, Vusi Ntlebi, Natasha Sanabria, Jitcy Joseph, Melissa Vetten. 'National Institute For Occupational Health, South Africa

10.1136/OEM-2021-EPI.369
Introduction Medical laboratory workers are exposed to COVID-19 in the community and through their interaction with samples received for testing. The National Health Laboratory service in South Africa serves $80 \%$ of the population providing medical tests. Information on all staff cases was collected in the Occupational Health and Safety Information System.

Methods Surveillance data from the OHASIS system was extracted from 01 April 2020-30 March 2021. All staff with a laboratory-confirmed positive test for SARS-COVID-19 were included in the study. NHLS staff had increased access to testing compared to the general public. An epidemic curve was plotted and compared to that for the country along with descriptive statistics.

Results A high proportion of NHLS staff tested positive for SARS Cov 2, 25.7\%. This varied across occupation groups with more educated occupations such as pathologists at less risk of COVID-19 compared to messengers and laboratory clerks. The epidemic curve for the facility peaked higher in the first wave compared to the rest of the country.

Conclusion The prevalence found in the laboratory staff may be a proxy for the country prevalence of COVID-19 if more access to testing had been available. The lower rate of positive cases in more educated staff may indicate the role of education in adherence to COVID-19 prevention measures.

\section{RF-453 SCORPION STING IN DOMESTIC SERVICE WORKERS (HOMEMAKERS) IN BRAZIL: AN EPIDEMIOLOGICAL APPROACH}

Ana Caroline Caldas Almeida, Yukari Figueroa Mise. 'Universidade Federal da Bahia, Brazil

\subsection{6/OEM-2021-EPI.370}

Introduction Scorpion stings (scorpionism) have common occurrence in home areas, which may predispose workers who perform domestic services, such as homemakers.

Objective To describe the incidence and clinical-epidemiological characteristics of cases of scorpionism in homemakers notified in Brazil, 2007-2019.

Methods Incident case study, individual, with 84,538 homemakers stung by scorpion, with economically active age (1865 years), occurred in Brazil from 2007-2019 and notified to the National System of Notifiable Diseases (SINAN). Cumulative incidence coefficients of scorpionism were estimated specifically for the female population occupied in domestic services. Demographic, socioeconomic and clinical-epidemiological characteristics were also investigated, using descriptive analysis.

Results The cumulative incidence of scorpionism was 110.9 cases/100,000 homemakers, 2007-2019, in Brazil. This scorpionism incidence in homemakers increased $349.7 \%$ in this period (48.7/100,000 homemakers to 219.0/100,000 homemakers) and was higher in the Brazilian Northeast (195.6/100,000 homemakers) and in the states of Alagoas (851.4 cases/100,000 homemakers), followed by Rio Grande do Norte (488.4/100,000 homemakers). Considering sociodemographic characteristics, the average age was 41 years old and occurred predominantly among workers with low education up to a maximum of elementary school (46.8\%), brown color (55.0\%), who were injured in an 
urban area $(72.3 \%)$, in the upper limbs (51.2\%) and who arrived early $(<3 \mathrm{~h})$ to the health service $(83.1 \%)$ after the injury. Poisonings were predominantly mild (92.1\%), and the relationship with work was not recognized by the service $(95.7 \%)$.

Conclusions Homemakers constitute a scorpionism risk population in Brazil. It is necessary to give more visibility to this problem from the perspective of the workers health.

\section{Exposure Assessment}

\section{RF-88 EVALUATING APPROACHES DESIGNED TO INCREASE SENSITIVITY OF CAPTURING WORK TASK INFORMATION IN A MULTI-CENTER HOSPITAL-BASED CASE-CONTROL STUDY IN ASIA}

'Sarah Locke, Melissa Friesen, Calvin Ge, Bryan Bassig, Wei Hu, Qing Lan, Nathaniel Rothman, Roel Vermeulen. 'U.S. National Cancer Institute, United States

\subsection{6/OEM-2021-EPI.371}

Objective Case-control studies use job- and industry-specific modules to systematically obtain details about the subjects' work tasks. This reduces respondent burden over asking the same questions for every job but relies on accurate module assignment to ensure relevant questions are asked.

Methods In a multi-center hospital-based case-control study of lymphoid and myeloid malignancies in Asia, lifetime occupational histories were collected for every job held by a subject. It comprised open-ended job title, work tasks, employer and its product/services, and yes/no screening questions pertaining to paints/stains, solvents/glues/degreasing agents, and engineered woods. These responses were used in a keywordbased algorithm to automatically assign each job to one of 23 modules, including 'generic exposure' and 'work location' modules. We evaluated the proportion of jobs assigned the work location module that were redirected to more specific modules and the prevalence of 'yes' responses to solventexposed tasks in the generic exposure module as indirect measures of the sensitivity of capturing relevant work task information.

Results The work location (29\%) and generic exposure $(21 \%)$ modules were the most frequently assigned to the 31,398 jobs. Of those jobs assigned the work location module, $8 \%$ were redirected to the generic module based on location of 'construction' and $12 \%$ were redirected to other modules. In total, 7,359 (23\%) jobs were assigned the generic module: $38 \%$ based on screening questions with no relevant keywords identified; $50 \%$ based on ambiguous keywords; and 11\% based on work location module. For these three pathways, the prevalence of 'yes' responses to any of the module's task and bystander questions were $77 \%, 44 \%$, and $23 \%$, respectively.

Conclusions The screening and the work location questions were successful in identifying jobs involved in tasks of interest that would have been missed if we had solely used the algorithm keyword search for jobs, tasks, and industries of interest.

\section{RF-170 MULTIFACTORIAL APPROACH FOR ASSESSING WORK ABILITY AMONG NURSING STAFF IN MONASTIR TEACHING HOSPITAL, TUNISIA}

Irtyah Merchaoui, 'Marouen Hayouni, Ines Rassas, Charfeddine Amri, Adnene Hanchi, Mohamed Akrout, Lamia Bouzgarrou, Jaques Malchaire, Neila Chaari. 'University of Monastir, Tunisia

\subsection{6/OEM-2021-EPI.372}

Introduction Work ability is a multifactorial concept that represents the interaction between human resources and working conditions. It is a dynamic process widely changing during human work life.

Objectives To measure the work ability index among nurses and to assess its determinants and propose strategies to maintain good work ability among this category of workers.

Methods This is a cross-sectional study conducted among a teaching hospital nurses between October 2017 and December 2018. A stratified random sampling method was employed, according to work department and gender to ensure a representative sample of the nurses working in the hospital. Nurses' work ability was assessed using the work ability index questionnaire including subjective and objective assessments. The job content questionnaire was used to assess job stress and social functioning at work for each participant. Each participant, including physical exercise performed out of work, and homework, underwent a battery of physical functional tests.

Results The mean work ability index score was 41.5 5.6. Multiple linear regression analysis revealed a positive significant association between mean work ability index score and regular nightshift working schedule, higher decision latitude, stronger grip and a better score of sit-to-stand test. In contrast, male gender, higher body mass index and higher level of perceived stress were inversely associated to the mean work ability index score.

Conclusion Efficient preventive actions based on a higher autonomy in nurses, promoting healthy lifestyles and introducing changes regarding schedule distribution according to the work ability index score would be helpful to promote work ability among nurses. The grip strength test and sit-to-stand test measures, seems to be suitable for nurses' work ability evaluation by occupational physician.

\section{RF-367 OCCUPATIONAL EXPOSURE TO AGENTS AND SUBSTANCES IN THE CARTAGENE COHORT}

${ }^{1}$ Nolwenn Noisel, Romain Pasquet, Lesley Richardson, Jack Siemiatycki, Philippe Broet. ${ }^{1}$ University of Montreal, Canada

\subsection{6/OEM-2021-EPI.373}

Introduction Occupational exposures are related to occupational diseases burden and increased susceptibility to health issues. The joint assessment of occupational exposure and disease outcome is the key to accelerate breakthroughs in occupational health research.

Objectives Occupational data including coding of occupations and exposure assessment from a large population cohort such as CARTaGENE may help the research community in uncovering workplace-related health disparities. 